\title{
Gravitational waves and spinning test particles
}

\author{
M. Mohseni* and H. R. Sepangi ${ }^{\dagger}$ \\ Physics Department, Shahid Beheshti University, Evin, Tehran 19839, Iran
}

October 29, 2018

\begin{abstract}
The motion of a classical spinning test particle in the field of a weak plane gravitational wave is studied. It is found that the characteristic dimensions of the particle's orbit is sensitive to the ratio of the spin to the mass of the particle. The results are compared with the corresponding motion of a particle without spin.
\end{abstract}

PACS: 04.20.-q, 04.25.-g, 04.30.-Nk

Keywords: spinning particles, gravitational waves, Dixon equations

\section{Introduction}

One of the problems of great importance in contemporary physics is the detection of gravitational waves which would profoundly affect both physics and observational astronomy [1]. Like other kind of waves, one may think of gravitational waves as wiggles propagating in some background. In fact these waves are wiggles of curvature propagating in a fixed background with the speed of light. The corresponding space-time metric may be written as $g_{\mu \nu}=g_{\mu \nu}^{0}+h_{\mu \nu}$ in which $g_{\mu \nu}^{0}$ stands for the background metric and $h_{\mu \nu}$ represents the gravitational wave field. In practical situations where the detection of these waves is our prime concern, one needs to consider only weak, plane, linear, waves in an essentially flat background because the amplitude of the waves crossing the solar system are very small even though the energy flux may be enormous [2]. We are thus led to the decomposition of the space-time metric as $g_{\mu \nu}=\eta_{\mu \nu}+h_{\mu \nu}$ where $\eta_{\mu \nu}=(-,+,+,+)$ is the usual Minkowski metric and $\left|h_{\mu \nu}\right| \ll 1$. It then follows from general relativity that $h_{\mu \nu}$ satisfies a generalized wave equation in three dimensions and there are only two components for $h_{\mu \nu}$ corresponding to two independent polarizations of gravitational waves usually denoted by $h_{+}$and $h_{\times}$[3]. Polarization of these waves contains valuable information about the sources generating them [1].

Gravitational waves alter the curvature of the region of the space-time they cross and hence affect the motion of particles. This problem has been studied extensively in the past e.g. see [2]-[5]. The basic idea is that free particles move on the geodesics of the basic field $g_{\mu \nu}$. The physical effect of a passing gravitational wave is that particles acquire a relative acceleration during the passage of the wave and hence gain a relative velocity after the wave has passed. The measurement of this relative velocity would hopefully provide a way to detect gravitational waves.

Grishchuck [6] studied the problem of the motion of a particle in the field of weak plane gravitational waves with different polarizations and calculated the trajectory of the particle as viewed

*email: mohseni@cc.sbu.ac.ir

${ }^{\dagger}$ email: hr-sepangi@cc.sbu.ac.ir 
from a locally inertial frame, that is, from the reference frame attached to a nearby particle. These trajectories, when projected onto the plane perpendicular to the direction of the wave propagation, become ellipsis or segments of straight lines whose dimension and orientation depend on the initial position of the particle and the polarization of the wave. By a suitable combination of the particle's initial position and wavelength of the wave, it is possible to find invariant dimensionless parameters which characterize the dimension of the trajectories.

In this paper we consider the problem of the determination of the motion of a classical free spinning particle in the field of a weak, plane gravitational wave with arbitrary polarizations. Here, several interesting phenomena will appear. The particle's spin couples to gravity and this makes the spin dynamical. Also, the trajectory of the particle deviates from geodesics of the field $g_{\mu \nu}$ as a result of a Lorentz-like force which originates from the spin-gravity coupling. This problem was previously considered from a different point of view in [7] where a supplementary equation was proposed causing the spinning particle to move along a geodesic.

In what follows we first review the equations of motion of a spinning particle in a general spacetime, sometimes known as Dixon's equations [13], and derive their weak field approximation. We then apply them to the case of a spinning test particle in the field of a gravitational wave and compare the results with those of a test particle without spin.

\section{Dixon's equations}

The motion of an extended body in a given background may be studied by treating the body via a multipole expansion. This expansion is based on the following procedure. The body sweeps out a narrow tube in space-time as it moves. Let $L$ be a line inside this tube representing the motion of the body. Denote the coordinates of the points on this line by $X^{\alpha}$ and define $\delta x^{\alpha}=X^{\alpha}-x^{\alpha}$, with $x^{\alpha}$ being the coordinates of the points of the body. Let $T_{\mu \nu}$ be the energy-momentum tensor describing the body and consider the quantities $\int T^{\mu \nu} d V, \int \delta x^{\lambda} T^{\mu \nu} d V, \int \delta x^{\lambda} \delta x^{\rho} T^{\mu \nu} d V, \cdots$ where the integrations are carried out on the three-dimensional hypersurfaces of fixed time $X^{0}=$ const.. These are in fact the successive terms in the above mentioned multipole expansion. If we only keep the first term, that is, if we assume that all the integrals containing $\delta x^{\mu}$ vanish, this will describe a single-pole particle. Similarly, we may consider a pole-dipole particle for which all the integrals with more than one factor of $\delta x^{\mu}$ vanish. Higher order approximations may be defined in a similar way. In the limit of $\left\|\delta x^{\alpha}\right\| \rightarrow 0$ with $\|$.$\| denoting the norm with respect to the induced metric on the three-dimensional$ hypersurface of constant time, the approximation becomes exact. Thus a single-pole particle is in fact a test particle without any internal structure. A pole-dipole particle is a test particle whose internal structure is expressed by its spin, an antisymmetric second rank tensor defined by

$$
s^{\mu \nu} \equiv \int\left(\delta x^{\mu} T^{0 \nu}-\delta x^{\nu} T^{0 \mu}\right) d V .
$$

The equations of motion are then obtained by applying Einstein's field equations together with the conservation of the energy-momentum tensor, $\nabla_{\mu} T^{\mu \nu}=0$, describing the body. For a single-pole this leads to a free particle moving along the geodesics associated with $g_{\mu \nu}$. This is the same as that obtained by Einstein and co-workers, who only made use of the field equations without resorting to the dynamical equation. Using the above procedure, Papapetrou [8] derived two sets of equations for the motion of a pole-dipole particle and its spin

$$
\dot{p}^{\mu}=-\frac{1}{2} R_{\nu \lambda \rho}^{\mu} v^{\nu} s^{\lambda \rho}
$$




$$
\dot{s}^{\mu \nu}=p^{\mu} v^{\nu}-p^{\nu} v^{\mu}
$$

in which $v^{\mu}=\frac{d X^{\mu}(\tau)}{d \tau}$ is the four-velocity of the particle along $L$ parameterized by $\tau$ with $v \cdot v=-1$, $R_{\nu \lambda \rho}^{\mu}$ is the curvature tensor, $p^{\mu}=\int T^{0 \mu} d V$ represents the particle's four-momentum along $L$ and

$$
\begin{aligned}
\dot{p}^{\mu} & \equiv \frac{d p^{\mu}(\tau)}{d \tau}+\Gamma_{\lambda \nu}^{\mu} v^{\lambda} p^{\nu} \\
\dot{s}^{\mu \nu} & \equiv \frac{d s^{\mu \nu}(\tau)}{d \tau}+\Gamma_{\lambda \rho}^{\mu} v^{\lambda} s^{\rho \nu}+\Gamma_{\lambda \rho}^{\nu} v^{\lambda} s^{\mu \rho}
\end{aligned}
$$

are covariant derivatives. Equation (11) expresses the coupling of the spin with curvature, and for either a flat space-time or a spinless particle reduces to the geodesic motion $\dot{p}^{\mu}=0$. Also, equation (2) is in fact an equation showing the balance of the particle and the field angular momenta. Unlike special relativity, $p^{\mu}$ and $v^{\mu}$ are not generally proportional to each other. Equations (1) and (2) alone are not adequate to give a solution and three of the components of $s^{\mu \nu}$ remain undetermined by these equations. This may be regarded as a result of $L$ not being yet fixed. To remedy this, several supplementary conditions have been proposed. A widely used condition proposed by Pirani [9] is $v_{\mu} s^{\mu \nu}=0$. However, it was shown in the second reference in [10] that this condition leads to unphysical solutions where the particle can admit helical paths in flat space-times. Also, it does not uniquely specify a world line through the body [14]. Here, we adopt the following condition [10]

$$
p_{\mu} s^{\mu \nu}=0 \text {. }
$$

This choice does not suffer from the appearance of unphysical solutions mentioned above and has other advantages discussed in [11, 12] and [13]. Equations (11) and (2) were derived by Dixon [11, 12] in a covariant manner and equation (3) was adopted as the supplementary condition. These equations have also been studied from different angles in [14]. The post-Newtonian approximation of equations (11) and (2) together with $v_{\mu} s^{\mu \nu}=0$ as the supplementary equation was studied in 15. From Dixon's equations we deduce the following relations

$$
p^{\mu} p_{\mu}=\text { const. }=-m^{2}, \quad \frac{1}{2} s^{\mu \nu} s_{\mu \nu}=\text { const. }=s^{2} .
$$

Alternatively, equation (3) enables us to express the particle's spin by the four-vector

$$
s^{\mu} \equiv \frac{1}{2 m} \eta^{\mu \nu \lambda \kappa} p_{\kappa} s_{\nu \lambda}
$$

where $\eta^{\mu \nu \lambda \kappa}=\frac{1}{\sqrt{-g}} \epsilon^{\mu \nu \lambda \kappa}$ is the alternating tensor with $\epsilon^{0123}=1$ and $g$ is the determinant of the metric. It then follows that $p_{\mu} s^{\mu}=0$ and $s^{\mu} s_{\mu}=s^{2}$. Under certain conditions [16], Dixon's equations may be thought of as the classical limit of the Dirac equation in curved space-time.

To find the particle's trajectory it is necessary to first find its four-velocity. Since there is no equations of motion for this purpose, one has to find the velocity from Dixon's equations in an indirect way. One such relation is [17]

$$
(p \cdot p) v^{\mu}=(p \cdot v)\left(p^{\mu}-\frac{2 R_{\sigma \nu \lambda \rho} p^{\nu} s^{\mu \sigma} s^{\lambda \rho}}{4 p \cdot p-R_{\mu \nu \lambda \kappa} s^{\mu \nu} s^{\lambda \kappa}}\right) .
$$

Unlike for $p^{\mu}$ there is no guarantee from the equations of motion that $v^{\mu}$ remains time-like. In fact Tod et.al. 17] have solved Dixon's equations for the case of the motion of a spinning test particle 
in the equatorial plane of a Kerr black-hole with the particle's spin perpendicular to this plane and found that there are orbits for which $v^{\mu}$ becomes null or space-like.

As Dixon's equations are reparametrization invariant, we have to fix a gauge before embarking on finding solutions for them. It is convenient to choose

$$
\frac{1}{m} p \cdot v=-1
$$

This is the gauge in which the instantaneous zero-momentum and zero-velocity frames are simultaneous [12].

\section{Spinning particles in a weak field}

It is generally difficult to solve equations (11)-(3) analytically and to our knowledge, the only solution that has been found up to now is the previously mentioned solution by Tod et.al. However, for the case of weak fields in a flat background where the metric is of the form $g_{\mu \nu}=\eta_{\mu \nu}+h_{\mu \nu}$, it is natural to assume that the change in the particle's velocity and spin are of the same order as $h_{\mu \nu}$. Therefore, we may decompose the particle's four-momentum, velocity and spin tensor as $p^{\mu}=p_{0}^{\mu}+\pi^{\mu}, v^{\mu}=v_{0}^{\mu}+w^{\mu}$ and $s^{\mu \nu}=s_{0}^{\mu \nu}+\sigma^{\mu \nu}$ with $\pi^{\mu}$, w $w^{\mu}$ and $\sigma^{\mu}$ being quantities of the same order as $h_{\mu \nu}$ and $p_{0}^{\mu}$, $v_{0}^{\mu}$ and $s_{0}^{\mu \nu}$ are the initial (before the passage of the wave) four-momentum, four-velocity and spin tensor of the particle respectively. The particle's trajectory can then be found from $\frac{d X^{\mu}(\tau)}{d \tau}=v^{\mu}$ subject to suitable initial conditions. Similarly we may express the particle's spin vector as $s^{\mu}=s_{0}^{\mu}+\sigma^{\mu}$. In terms of these decompositions, the zeroth order equations become

$$
\begin{gathered}
\frac{d p_{0}^{\mu}}{d \tau}=\frac{d s_{0}^{\mu \nu}}{d \tau}=0 \\
p_{\mu_{0}} s_{0}^{\mu \nu}=0 \\
s_{0}^{\mu}=\frac{1}{2 m} \epsilon_{\nu \lambda \kappa}^{\mu} v_{0}^{\kappa} s_{0}^{\nu \lambda} .
\end{gathered}
$$

Equation (5) is trivial: in the absence of any field the particle's four momentum and spin tensor are conserved. Equation (6) eliminates three of the components of $s^{\mu \nu}$ and equation (7) may be used to express $s_{0}^{\mu \nu}$ in terms of $s_{0}^{\mu}$. For example, for $p_{0}^{\mu}=(m, 0,0,0)$ these lead to $s_{0}^{0 \mu}=0$ and $s_{0}^{12}=s_{0}^{3}, s_{0}^{13}=-s_{0}^{2}, s_{0}^{23}=s_{0}^{1}$.

For the metric $g_{\mu \nu}=g_{\mu \nu}^{0}+h_{\mu \nu}$, the curvature tensor and the connection may be written as

$$
R_{\mu \nu \kappa \lambda}=R_{\mu \nu \kappa \lambda}^{(0)}+r_{\mu \nu \kappa \lambda} \text { and } \Gamma_{\nu \kappa}^{\mu}=\Gamma_{\nu \kappa}^{(0) \mu}+\gamma_{\nu \kappa}^{\mu} .
$$

However, the background quantities $R_{\mu \nu \kappa \lambda}^{(0)}$ and $\Gamma_{\nu \kappa}^{(0) \mu}$ vanish for the flat background and the first order form of equations (1), (2) and (田) reads

$$
\begin{aligned}
\frac{d \pi^{\mu}(\tau)}{d \tau} & =-\frac{1}{2} r_{\rho \lambda \nu}^{\mu} v_{0}^{\rho} s_{0}^{\lambda \nu}-\gamma_{\lambda \nu}^{\mu} v_{0}^{\lambda} p_{0}^{\nu} \\
\frac{d \sigma^{\mu \nu}(\tau)}{d \tau} & =-\frac{1}{2 m} r_{\rho \sigma \kappa \lambda} v_{0}^{\nu} v_{0}^{\sigma} s_{0}^{\mu \rho} s_{0}^{\kappa \lambda}-\gamma_{\lambda \rho}^{\mu} v_{0}^{\lambda} s_{0}^{\rho \nu}-\mu \leftrightarrow \nu \\
w^{\mu}(\tau) & =\frac{1}{m} \pi^{\mu}(\tau)+\frac{1}{2 m^{2}} r_{\kappa \lambda \rho \sigma} v_{0}^{\lambda} s_{0}^{\mu \kappa} s_{0}^{\rho \sigma}
\end{aligned}
$$


with the supplementary equation written as

$$
\eta_{\mu \lambda}\left(s_{0}^{\mu \nu} \pi^{\lambda}(\tau)+p_{0}^{\lambda} \sigma^{\mu \nu}(\tau)\right)+h_{\mu \lambda} p_{0}^{\lambda} s_{0}^{\mu \nu}=0 .
$$

The corresponding expression for $\sigma^{\mu}$ is

$$
\sigma^{\mu}(\tau)=\frac{1}{2 m} \epsilon^{\mu \lambda \rho}{ }_{\nu \rho}^{\rho}\left(p_{0}^{\rho} \sigma^{\nu \lambda}+\pi^{\rho} s_{0}^{\nu \lambda}+2 p_{0}^{\rho} s_{0}{ }^{\kappa}{ }^{\nu}{ }^{\nu}{ }_{\kappa}+p_{0}^{\kappa} s_{0}^{\nu \lambda} h^{\rho}{ }_{\kappa}\right) .
$$

Therefore, given an initial data set $v_{0}^{\mu}=\left(v_{0}^{0}, v_{0}^{1}, v_{0}^{2}, v_{0}^{3}\right)$ and $s_{0}^{\mu}=\left(s_{0}^{0}, s_{0}^{1}, s_{0}^{2}, s_{0}^{3}\right)$, the orbit of the particle is given by

$$
X^{\mu}(\tau)=X_{0}^{\mu}(\tau)+\chi^{\mu}(\tau)
$$

and its spin components are determined by solving equations (8)-(11) subject to suitable initial conditions. Here, $\frac{d X_{0}^{\mu}(\tau)}{d \tau}=v_{0}^{\mu}$ and $\frac{d \chi^{\mu}(\tau)}{d \tau}=w^{\mu}$.

\section{Gravitational waves}

In this section we apply the procedure described in the previous section to a spinning particle in the field of a weak plane gravitational wave propagating in the $z$-direction in some coordinate system. For such a field, the metric may be written as [6]

$$
d s^{2}=-d t^{2}+(1+a(t-z)) d x^{2}+(1-a(t-z)) d y^{2}+2 b(t-z) d x d y+d z^{2}
$$

with $a(t-z)=h_{+} \sin (\omega(t-z))$ and $b(t-z)=h_{\times} \cos (\omega(t-z))$. A wave with linear polarization is described by $h_{+}=0$ or $h_{\times}=0$ and a circularly polarized wave is characterized by $h_{+}= \pm h_{\times}$.

For this metric, it is straightforward but lengthy to solve the relevant equations. The resulting expressions for the trajectory of the particle and the evolution of its spin are too complicated to be included here, but are given for $\chi^{\mu}(\tau)$ in the Appendix. However, they become manageable for the physically interesting case of the motion of a particle initially at rest with respect to the coordinate system in which the metric (14) is expressed. In this frame, $v_{0}^{0}=1, v_{0}^{1}=v_{0}^{2}=v_{0}^{3}=0$ and an initial spin orientation may be taken as $\left(0, s_{0}^{1}, s_{0}^{2}, s_{0}^{3}\right)$. For such a particle the trajectory (with respect to the coordinate system $(t, x, y, z)$ and the initial conditions $\left.\chi^{\mu}(0)=w^{\mu}(0)=\sigma^{\mu}(0)=0\right)$ is given by

$$
\begin{aligned}
& t(\tau)=\tau \\
& x(\tau)=-\frac{1}{2 m^{2}} s_{0}^{2}\left(s_{0}^{3} \omega h_{\times}+m h_{+}\right)(\omega \tau-\sin (\omega \tau))+\frac{1}{2 m^{2}} s_{0}^{1}\left(s_{0}^{3} \omega h_{+}+m h_{\times}\right)(1-\cos (\omega \tau)) \\
& y(\tau)=-\frac{1}{2 m^{2}} s_{0}^{1}\left(s_{0}^{3} \omega h_{\times}+m h_{+}\right)(\omega \tau-\sin (\omega \tau))-\frac{1}{2 m^{2}} s_{0}^{2}\left(s_{0}^{3} \omega h_{+}+m h_{\times}\right)(1-\cos (\omega \tau)) \\
& z(\tau)=\frac{1}{m^{2}} s_{0}^{1} s_{0}^{2} \omega h_{\times}(\omega \tau-\sin (\omega \tau))+\frac{1}{2 m^{2}} \omega h_{+}\left(\left(s_{0}^{2}\right)^{2}-\left(s_{0}^{1}\right)^{2}\right)(1-\cos (\omega \tau))
\end{aligned}
$$

and the equations for spin evolution are

$$
\begin{aligned}
& s^{0}(\tau)=\frac{\omega}{2 m} h_{\times}\left(\left(s_{0}^{1}\right)^{2}-\left(s_{0}^{2}\right)^{2}\right) \sin (\omega \tau)-\frac{\omega}{m} s_{0}^{1} s_{0}^{2} h_{+}(1-\cos (\omega \tau)) \\
& s^{1}(\tau)=s_{0}^{1}-\frac{1}{2} s_{0}^{1} h_{+} \sin (\omega \tau)+\frac{1}{2} s_{0}^{2} h_{\times}(1-\cos (\omega \tau)) \\
& s^{2}(\tau)=s_{0}^{2}+\frac{1}{2} s_{0}^{2} h_{+} \sin (\omega \tau)+\frac{1}{2} s_{0}^{1} h_{\times}(1-\cos (\omega \tau)) \\
& s^{3}(\tau)=s_{0}^{3} .
\end{aligned}
$$


We can use equations (20)-(22) to study the evolution of the particle's spin. From these equations it is clear that the spin evolution does not depend on $s_{0}^{3}$, nor is this component affected by the wave. Therefore, the initial component of the spin along the direction of the wave propagation is unaffected by the wave. The components $s^{1}(\tau)$ and $s^{2}(\tau)$ depend on both $s_{0}^{1}$ and $s_{0}^{2}$ giving rise to the spin vector precessing about the $z$-axis. The rotation of the spin vector is very small and as equations (20-21) show, they are of the order of $h_{+} s$ or $h_{\times} s$, where $s$ is the relevant component of the spin. The appearance of a nonzero $s_{0}^{0}$ component is related to the fact that the particle is not at rest with respect to this coordinate system.

The particle's trajectory with respect to the frame $(t, x, y, z)$ can be determined from equations (15)-(18). Putting $s_{0}^{1}=s_{0}^{2}=0$ and $s_{0}^{3}=s$ in these equations result in

$$
(t(\tau), x(\tau), y(\tau), z(\tau))=(\tau, 0,0,0)
$$

which means that the particle remains at rest at its initial position. This behaviour is the same as that of a particle without spin. This has no invariant geometrical meaning and may be interpreted as follows: the coordinate system $(t, x, y, z)$ is the one always remaining attached to the particle. The physical effect of the wave is on the relative motion of the particles. To study this we switch to $(\bar{t}, \bar{x}, \bar{y}, \bar{z})$, a locally inertial frame associated with the worldline $x(\tau)=y(\tau)=z(\tau)=0$ given by [6]

$$
\begin{aligned}
& \bar{t}=t-\frac{1}{4} \frac{\partial a}{\partial t}\left(y^{2}-x^{2}\right)-\frac{\partial b}{\partial t} x y \\
& \bar{x}=x+\frac{1}{2} a x-\frac{1}{2} b y-\frac{1}{2} \frac{\partial a}{\partial t} x z+\frac{1}{2} \frac{\partial b}{\partial t} y z \\
& \bar{y}=y-\frac{1}{2} a y-\frac{1}{2} b x-\frac{1}{2} \frac{\partial a}{\partial t} y z+\frac{1}{2} \frac{\partial b}{\partial t} x z \\
& \bar{z}=z-\frac{1}{4} \frac{\partial a}{\partial t}\left(y^{2}-x^{2}\right)-\frac{\partial b}{\partial t} x y
\end{aligned}
$$

with $a, b, \partial a / \partial t$, and $\partial b / \partial t$ being evaluated at $x=y=z=0$. In this way, we are able to study the relative motion of two nearby particles, one with spin and the other without spin.

The motion of a nearby particle without spin initially at the position $\left(l_{1}, l_{2}, 0\right)$ with respect to this frame was studied in [19]. The motion of a spinning particle with its spin parallel to the direction of the wave propagation with respect to this frame is the same as a particle without spin. Let us now consider the motion of a particle with its spin perpendicular to the $z$-axis. We thus set, say, $s_{0}^{1}=s$ and $s_{0}^{2}=s_{0}^{3}=0$ with the initial position $x=l_{1}, y=l_{2}, z=0$. We then obtain from equations (15)-(18) and (24)-(27)

$$
\begin{aligned}
& \bar{t}(\tau)=\tau-\frac{\omega h_{+}}{4}\left(l_{2}^{2}-l_{1}^{2}\right) \cos (\omega \tau)+\frac{\omega h_{\times}}{2} l_{1} l_{2} \sin (\omega \tau) \\
& \bar{x}(\tau)=l_{1}\left(1+\frac{h_{+}}{2} \sin (\omega \tau)\right)-\frac{h_{\times}}{2} l_{2} \cos (\omega \tau)+\frac{s h_{\times}}{2 m}(1-\cos (\omega \tau)) \\
& \bar{y}(\tau)=l_{2}\left(1-\frac{h_{+}}{2} \sin (\omega \tau)\right)-\frac{h_{\times}}{2} l_{1} \cos (\omega \tau)-\frac{s h_{+}}{2 m}(\omega \tau-\sin (\omega \tau)) \\
& \bar{z}(\tau)=-\frac{\omega h_{+}}{4}\left(l_{2}^{2}-l_{1}^{2}\right) \cos (\omega \tau)-\frac{\omega h_{\times}}{2} l_{1} l_{2} \sin (\omega \tau)-\frac{\omega h_{+}}{2}\left(\frac{s}{m}\right)^{2}(1-\cos (\omega \tau)) .
\end{aligned}
$$

This set of equations determines the trajectory of a spinning particle initially at rest at the point $\left(l_{1}, l_{2}, 0\right)$ with respect to a locally inertial frame associated with the origin. In general, this trajectory is in the form of a helix whose size is sensitive to the ratio of the particle's spin to its mass. In figure 1 the trajectories of a spinning particle and that of a particle without spin are shown for $\frac{s}{m} \sim 10^{-5}$ 
$\mathrm{m}$. We note that in a system of units in which $c=1$, this ratio for an electron is of the order of $10^{-13} \mathrm{~m}$ and for an object like the Earth is $10^{-1} \mathrm{~m}$. For $\frac{s}{m} \sim \omega^{-1} \sim \lambda$ the spin contribution to the displacement in the $z$-direction is of the same order as those for the $x$ and $y$-directions. Also, it is worth mentioning that for $\frac{s}{m} \sim l_{1} \sim l_{2}$, the contribution of the terms involving the spin in equations (29)-(31) are as important as the terms representing the motion of a spinless particle. Therefore, the ratio $\frac{s}{m \lambda}$ seems to play a remarkable role in this solution.

One may estimate the displacement of a test particle using equations (29)-(31). During one period of the wave, i.e. $\omega \tau=2 \pi$, a particle initially at $\left(l_{1}, l_{2}, 0\right)$ would reach the space-time point

$$
\begin{aligned}
\bar{t} & =\tau-\frac{1}{4} \omega h_{+}\left(l_{2}^{2}-l_{1}^{2}\right) \\
\bar{x} & =l_{1}-\frac{1}{2} h_{\times} l_{2} \\
\bar{y} & =l_{2}-\frac{1}{2} h_{\times} l_{1}-\pi h_{+} \frac{s}{m} \\
\bar{z} & =-\frac{1}{4} h_{+} \omega\left(l_{2}^{2}-l_{1}^{2}\right) .
\end{aligned}
$$

corresponding to a displacement of the order of $h l$ with $l=\sqrt{l_{1}^{2}+l_{2}^{2}}$. Choosing the set of parameters $\omega \sim 10^{3} \mathrm{~Hz}, l_{1} \sim l_{2} \sim \frac{s}{m} \sim 10^{-5} \mathrm{~m}$ and $h_{+} \sim h_{\times} \sim 10^{-21}$, the displacement would be of the order of $10^{-26} \mathrm{~m}$. The above values for $\omega$ and $h_{+}, h_{\times}$stem from the expectation that for the gravitational waves accessible to earth based detectors $\omega \sim 1-10^{4} \mathrm{~Hz}$ with a typical amplitude of $h \sim 10^{-21}$ [何]. This value for $\omega$ together with $\omega \tau=2 \pi$ corresponds to $\tau \sim 10^{-3}$ sec which is also a typical duration for short bursts of gravitational waves [2].

One would hope to be able to detect such gravitational waves by the future sensitive detectors such as LIGO [1] currently under construction. In general, these displacements are small but could be cumulative and hence likely to be observed.

\section{Conclusions}

We have studied the motion of spinning particles in the field of gravitational waves. The solutions we have found are free of the problems mentioned before, namely, the violation of the normalization condition $v \cdot v=-1$. For a spinless particle, the velocity and hence its orbit depends on its initial position. For spinning particles, there is a contribution to the change in the trajectory from the coupling of the spin to the curvature. This is independent of the initial position and rather depends on the initial orientation of the spin. The amount of deviation from the geodesic path depends on the ratio $\frac{s}{m \lambda}$. For a certain value of this ratio, the displacement caused by the spin-gravity coupling could be as important as that caused by the usual tidal force of gravity waves. Although the particle's displacement is very small, one may hope to have it amplified in the course of the wave passage, e.g., by a suitable arrangement of reflectors.

In this paper we have only considered monochromatic waves i.e. those with a single $\omega$. In principle it is possible to generalize this procedure to the case of gravitational waves which are not monochromatic [5]. Our approximate solutions may also provide some hints for finding exact solutions to Dixon's equations for a general plane wave. For example, we can investigate whether there are exact solutions to these equations corresponding to the motion in certain planes with specific spin orientation. Indeed, equation (23) is such an exact solution. This has subsequently been verified in [18] where a class of exact solutions along whose path the initial orientation of the spin of the particle 


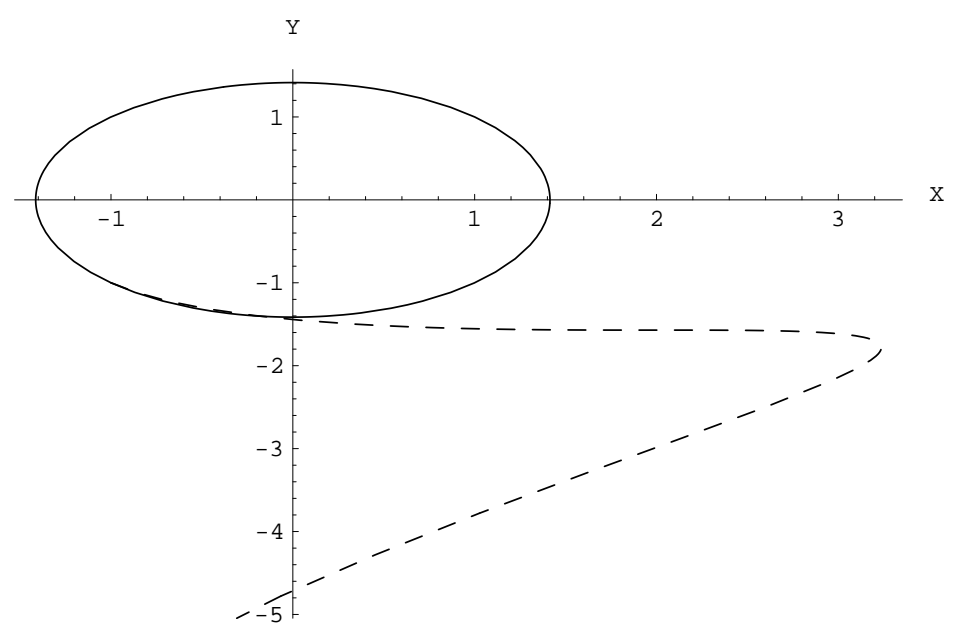

Figure 1: The path of a spinless particle (solid line) and a spinning particle (dashed line) in the field of a gravitational wave. They are originally located at the same position $(-1,-1)$. The values of the parameters used here are $\omega \sim 1 \mathrm{kHz}, h_{+} \sim h_{\times} \sim 10^{-21}, l_{1} \sim l_{2} \sim 10^{-5} \mathrm{~m}$ and $\frac{s}{m} \sim 10^{-5} \mathrm{~m}$. For convenience, we have adopted a system of coordinates given by $X=10^{26}\left(\bar{x}-10^{-5}\right)$ and $Y=$ $10^{26}\left(\bar{y}-10^{-5}\right)$. The displacements in the $Z$ direction are very small and thus are not shown in the figure.

remains unchanged, is presented. It should also be interesting to study the cumulative effects of the waves on the motion of particles [19].

It is worth mentioning that within the context of the perturbative framework presented here, we have also used Tulczyjew's description (a special case of Papapetrou equations together with Pirani supplementary condition) [20]. We have found that in some special circumstances, e.g. when the test particle is initially at rest, the spin evolution is exactly the same for various supplementary conditions mentioned above. However, in general the trajectories turn out to be only slightly different. The general case of Papapetrou-Pirani will be reported elsewhere [21].

\section{Acknowledgements}

We would like to thank R. W. Tucker for introducing this problem to us. M. M. wishes to thank C. Wang for valuable comments. Both authors acknowledge useful discussions with S. S. Gousheh.

\section{Appendix}

In this appendix, we give the components of the trajectory of a test particle [22] in the field of a gravitational wave specified by the metric (14). To make the expressions manageable, the following substitutions have been made: $s_{1}, s_{2}, s_{3}, \rho, \kappa$ stand for $s_{0}^{1}, s_{0}^{2}, s_{0}^{3},(\alpha-\delta) \omega \tau-\sin ((\alpha-\delta) \omega \tau)$ and $1-\cos ((\alpha-\delta) \omega \tau)$ respectively with $\left(v_{0}^{0}, v_{0}^{1}, v_{0}^{2}, v_{0}^{3}\right)=(\alpha, \beta, \gamma, \delta)$. These components together with those of the spin (not presented here) satisfy equation (11). Also, using equation (13), they reduce to equations (15)-(18) for the set of initial parameters discussed in section 4.

$$
\begin{aligned}
\chi^{0}(\tau) & =\left\{\rho \left[\omega ^ { 2 } h _ { \times } \left(s_{1}^{2}\left(\alpha^{3} \beta \gamma-3 \alpha^{2} \beta \gamma \delta-\alpha \beta^{3} \gamma+\alpha \beta \gamma^{3}+3 \alpha \beta \gamma \delta^{2}+\beta^{3} \gamma \delta-\beta \gamma^{3} \delta-\beta \gamma \delta^{3}\right)\right.\right.\right. \\
& +s_{1} s_{2}\left(2 \alpha^{4} \delta-\alpha^{3} \beta^{2}-\alpha^{3} \gamma^{2}-6 \alpha^{3} \delta^{2}+\alpha^{2} \beta^{2} \delta+\alpha^{2} \gamma^{2} \delta+6 \alpha^{2} \delta^{3}+\alpha \beta^{4}-2 \alpha \beta^{2} \gamma^{2}+\alpha \beta^{2} \delta^{2}\right.
\end{aligned}
$$




$$
\begin{aligned}
& \left.+\alpha \gamma^{4}+\alpha \gamma^{2} \delta^{2}-2 \alpha \delta^{4}-\beta^{4} \delta+2 \beta^{2} \gamma^{2} \delta-\beta^{2} \delta^{3}-\gamma^{4} \delta-\gamma^{2} \delta^{3}\right)+s_{1} s_{3}\left(-\alpha^{4} \gamma+4 \alpha^{3} \gamma \delta\right. \\
& \left.+3 \alpha^{2} \beta^{2} \gamma-\alpha^{2} \gamma^{3}-6 \alpha^{2} \gamma \delta^{2}-6 \alpha \beta^{2} \gamma \delta+2 \alpha \gamma^{3} \delta+4 \alpha \gamma \delta^{3}+3 \beta^{2} \gamma \delta^{2}-\gamma^{3} \delta^{2}-\gamma \delta^{4}\right) \\
& +s_{2}^{2}\left(\alpha^{3} \beta \gamma-3 \alpha^{2} \beta \gamma \delta+\alpha \beta^{3} \gamma-\alpha \beta \gamma^{3}+3 \alpha \beta \gamma \delta^{2}-\beta^{3} \gamma \delta+\beta \gamma^{3} \delta-\beta \gamma \delta^{3}\right)+s_{2} s_{3}\left(-\alpha^{4} \beta\right. \\
& +4 \alpha^{3} \beta \delta-\alpha^{2} \beta^{3}+3 \alpha^{2} \beta \gamma^{2}-6 \alpha^{2} \beta \delta^{2}+2 \alpha \beta^{3} \delta-6 \alpha \beta \gamma^{2} \delta+4 \alpha \beta \delta^{3}-\beta^{3} \delta^{2}+3 \beta \gamma^{2} \delta^{2} \\
& \left.\left.-\beta \delta^{4}\right)+s_{3}^{2}\left(-2 \alpha^{3} \beta \gamma+6 \alpha^{2} \beta \gamma \delta-6 \alpha \beta \gamma \delta^{2}+2 \beta \gamma \delta^{3}\right)\right)+m h_{+} \omega\left(s_{1}\left(-\alpha^{2} \gamma+\alpha \gamma \delta+2 \beta^{2} \gamma\right)\right. \\
& \left.\left.+s_{2}\left(-\alpha^{2} \beta+\alpha \beta \delta+2 \beta \gamma^{2}\right)+s_{3}(-2 \alpha \beta \gamma+2 \beta \gamma \delta)\right)+2 m^{2} h_{\times} \alpha \beta \gamma\right]+\kappa\left[h _ { + } \omega ^ { 2 } \left(s _ { 1 } ^ { 2 } \left(-\alpha^{4} \delta\right.\right.\right. \\
& +\alpha^{3} \gamma^{2}+3 \alpha^{3} \delta^{2}+\alpha^{2} \beta^{2} \delta-2 \alpha^{2} \gamma^{2} \delta-3 \alpha^{2} \delta^{3}-2 \alpha \beta^{2} \gamma^{2}-2 \alpha \beta^{2} \delta^{2}+\alpha \gamma^{2} \delta^{2}+\alpha \delta^{4} \\
& \left.+2 \beta^{2} \gamma^{2} \delta+\beta^{2} \delta^{3}\right)+s_{1} s_{2}\left(2 \alpha \beta^{3} \gamma-2 \alpha \beta \gamma^{3}-2 \beta^{3} \gamma \delta+2 \beta \gamma^{3} \delta\right)+s_{1} s_{3}\left(\alpha^{4} \beta-4 \alpha^{3} \beta \delta\right. \\
& \left.-\alpha^{2} \beta^{3}+3 \alpha^{2} \beta \gamma^{2}+6 \alpha^{2} \beta \delta^{2}+2 \alpha \beta^{3} \delta-6 \alpha \beta \gamma^{2} \delta-4 \alpha \beta \delta^{3}-\beta^{3} \delta^{2}+3 \beta \gamma^{2} \delta^{2}+\beta \delta^{4}\right) \\
& +s_{2}^{2}\left(\alpha^{4} \delta-\alpha^{3} \beta^{2}-3 \alpha^{3} \delta^{2}+2 \alpha^{2} \beta^{2} \delta-\alpha^{2} \gamma^{2} \delta+3 \alpha^{2} \delta^{3}+2 \alpha \beta^{2} \gamma^{2}-\alpha \beta^{2} \delta^{2}+2 \alpha \gamma^{2} \delta^{2}\right. \\
& \left.-\alpha \delta^{4}-2 \beta^{2} \gamma^{2} \delta-\gamma^{2} \delta^{3}\right)+s_{2} s_{3}\left(-\alpha^{4} \gamma+4 \alpha^{3} \gamma \delta-3 \alpha^{2} \beta^{2} \gamma+\alpha^{2} \gamma^{3}-6 \alpha^{2} \gamma \delta^{2}+6 \alpha \beta^{2} \gamma \delta\right. \\
& \left.-2 \alpha \gamma^{3} \delta+4 \alpha \gamma \delta^{3}-3 \beta^{2} \gamma \delta^{2}+\gamma^{3} \delta^{2}-\gamma \delta^{4}\right)+s_{3}^{2}\left(\alpha^{3} \beta^{2}-\alpha^{3} \gamma^{2}-3 \alpha^{2} \beta^{2} \delta+3 \alpha^{2} \gamma^{2} \delta\right. \\
& \left.\left.+3 \alpha \beta^{2} \delta^{2}-3 \alpha \gamma^{2} \delta^{2}-\beta^{2} \delta^{3}+\gamma^{2} \delta^{3}\right)\right)+m \omega h_{\times}\left(s_{1}\left(\alpha^{2} \beta-\alpha \beta \delta-\beta^{3}+\beta \gamma^{2}\right)+s_{2}\left(-\alpha^{2} \gamma\right.\right. \\
& \left.\left.\left.\left.+\alpha \gamma \delta-\beta^{2} \gamma+\gamma^{3}\right)+s_{3}\left(\alpha \beta^{2}-\alpha \gamma^{2}-\beta^{2} \delta+\gamma^{2} \delta\right)\right)+m^{2} h_{+}\left(-\beta^{2}+\gamma^{2}\right)\right]\right\} /\left(2 m^{2} \omega \alpha(\alpha-\delta)\right) \\
& \chi^{1}(\tau)=\left\{\rho \left[\omega ^ { 2 } h _ { \times } \left(s_{1}^{2}\left(\alpha^{2} \beta^{2} \gamma-2 \alpha \beta^{2} \gamma \delta-\beta^{4} \gamma+\beta^{2} \gamma^{3}+\beta^{2} \gamma \delta^{2}\right)+s_{1} s_{2}\left(-\alpha^{4} \beta+3 \alpha^{3} \beta \delta\right.\right.\right.\right. \\
& \left.+\alpha^{2} \beta^{3}-\alpha^{2} \beta \gamma^{2}-3 \alpha^{2} \beta \delta^{2}-\alpha \beta^{3} \delta-\alpha \beta \gamma^{2} \delta+\alpha \beta \delta^{3}-2 \beta^{3} \gamma^{2}+2 \beta \gamma^{4}+2 \beta \gamma^{2} \delta^{2}\right) \\
& +s_{1} s_{3}\left(2 \alpha^{2} \beta \gamma \delta+2 \alpha \beta^{3} \gamma-2 \alpha \beta \gamma^{3}-4 \alpha \beta \gamma \delta^{2}-2 \beta^{3} \gamma \delta+2 \beta \gamma^{3} \delta+2 \beta \gamma \delta^{3}\right)+s_{2}^{2}\left(\alpha^{4} \gamma\right. \\
& \left.-\alpha^{3} \gamma \delta+\alpha^{2} \beta^{2} \gamma-2 \alpha^{2} \gamma^{3}-\alpha^{2} \gamma \delta^{2}-\alpha \beta^{2} \gamma \delta+\alpha \gamma^{3} \delta+\alpha \gamma \delta^{3}-\beta^{2} \gamma^{3}+\gamma^{5}+\gamma^{3} \delta^{2}\right) \\
& +s_{2} s_{3}\left(-\alpha^{5}+2 \alpha^{4} \delta-\alpha^{3} \beta^{2}+3 \alpha^{3} \gamma^{2}+2 \alpha^{2} \beta^{2} \delta-4 \alpha^{2} \gamma^{2} \delta-2 \alpha^{2} \delta^{3}+2 \alpha \beta^{2} \gamma^{2}-\alpha \beta^{2} \delta^{2}\right. \\
& \left.-2 \alpha \gamma^{4}-\alpha \gamma^{2} \delta^{2}+\alpha \delta^{4}-2 \beta^{2} \gamma^{2} \delta+2 \gamma^{4} \delta+2 \gamma^{2} \delta^{3}\right)+s_{3}^{2}\left(-\alpha^{4} \gamma+2 \alpha^{3} \gamma \delta-\alpha^{2} \beta^{2} \gamma+\alpha^{2} \gamma^{3}\right. \\
& \left.\left.+2 \alpha \beta^{2} \gamma \delta-2 \alpha \gamma^{3} \delta-2 \alpha \gamma \delta^{3}-\beta^{2} \gamma \delta^{2}+\gamma^{3} \delta^{2}+\gamma \delta^{4}\right)\right)+m h_{+} \omega\left(s_{1}(\alpha \beta \gamma)+s_{2}\left(-\alpha^{3}+\alpha^{2} \delta\right.\right. \\
& \left.\left.\left.+\alpha \gamma^{2}\right)+s_{3}\left(-\alpha^{2} \gamma+\alpha \gamma \delta\right)\right)+m^{2} h_{\times} \alpha^{2} \gamma\right]+\kappa\left[h _ { + } \omega ^ { 2 } \left(s _ { 1 } ^ { 2 } \left(-\alpha^{3} \beta \delta+\alpha^{2} \beta \gamma^{2}+2 \alpha^{2} \beta \delta^{2}\right.\right.\right. \\
& \left.+\alpha \beta^{3} \delta-\alpha \beta \gamma^{2} \delta-\alpha \beta \delta^{3}-2 \beta^{3} \gamma^{2}-\beta^{3} \delta^{2}\right)+s_{1} s_{2}\left(-\alpha^{4} \gamma+\alpha^{3} \gamma \delta+3 \alpha^{2} \beta^{2} \gamma+\alpha^{2} \gamma^{3}+\alpha^{2} \gamma \delta^{2}\right. \\
& \left.-\alpha \beta^{2} \gamma \delta-\alpha \gamma^{3} \delta-\alpha \gamma \delta^{3}-4 \beta^{2} \gamma^{3}-2 \beta^{2} \gamma \delta^{2}\right)+s_{1} s_{3}\left(\alpha^{5}-2 \alpha^{4} \delta-\alpha^{3} \beta^{2}-\alpha^{3} \gamma^{2}\right. \\
& \left.+2 \alpha^{2} \gamma^{2} \delta+2 \alpha^{2} \delta^{3}+4 \alpha \beta^{2} \gamma^{2}+3 \alpha \beta^{2} \delta^{2}-\alpha \gamma^{2} \delta^{2}-\alpha \delta^{4}-4 \beta^{2} \gamma^{2} \delta-2 \beta^{2} \delta^{3}\right)+s_{2}^{2}\left(-\alpha^{4} \beta\right. \\
& \left.+2 \alpha^{3} \beta \delta+3 \alpha^{2} \beta \gamma^{2}-\alpha^{2} \beta \delta^{2}-2 \alpha \beta \gamma^{2} \delta-2 \beta \gamma^{4}-\beta \gamma^{2} \delta^{2}\right)+s_{2} s_{3}\left(-4 \alpha^{3} \beta \gamma+6 \alpha^{2} \beta \gamma \delta+4 \alpha \beta \gamma^{3}\right. \\
& \left.\left.-4 \beta \gamma^{3} \delta-2 \beta \gamma \delta^{3}\right)+s_{3}^{2}\left(\alpha^{4} \beta-2 \alpha^{3} \beta \delta-2 \alpha^{2} \beta \gamma^{2}+4 \alpha \beta \gamma^{2} \delta+2 \alpha \beta \delta^{3}-2 \beta \gamma^{2} \delta^{2}-\beta \delta^{4}\right)\right) \\
& \left.\left.+m h_{\times} \omega\left(s_{1}\left(\alpha^{3}-\alpha^{2} \delta-\alpha \beta^{2}\right)+s_{2}(-\alpha \beta \gamma)+s_{3}\left(\alpha^{2} \beta-\alpha \beta \delta\right)\right)-m^{2} h_{+} \alpha^{2} \beta\right]\right\} /\left(2 m^{2} \omega \alpha^{2}\right) \\
& \chi^{2}(\tau)=-\chi^{1}\left(\tau \rightarrow-\tau, \beta \leftrightarrow \gamma, s_{1} \leftrightarrow s_{2}\right) \\
& \chi^{3}(\tau)=\left\{\rho \left[h _ { \times } \omega ^ { 2 } \left(s _ { 1 } ^ { 2 } \left(-\alpha^{4} \beta \gamma+3 \alpha^{3} \beta \gamma \delta+\alpha^{2} \beta^{3} \gamma+\alpha^{2} \beta \gamma^{3}-3 \alpha^{2} \beta \gamma \delta^{2}-3 \alpha \beta^{3} \gamma \delta-\alpha \beta \gamma^{3} \delta+\alpha \beta \gamma \delta^{3}\right.\right.\right.\right. \\
& \left.+2 \beta^{3} \gamma \delta^{2}\right)+s_{1} s_{2}\left(2 \alpha^{6}-6 \alpha^{5} \delta-3 \alpha^{4} \beta^{2}-3 \alpha^{4} \gamma^{2}+6 \alpha^{4} \delta^{2}+7 \alpha^{3} \beta^{2} \delta+7 \alpha^{3} \gamma^{2} \delta-2 \alpha^{3} \delta^{3}\right. \\
& +\alpha^{2} \beta^{4}+2 \alpha^{2} \beta^{2} \gamma^{2}-5 \alpha^{2} \beta^{2} \delta^{2}+\alpha^{2} \gamma^{4}-5 \alpha^{2} \gamma^{2} \delta^{2}-\alpha \beta^{4} \delta-6 \alpha \beta^{2} \gamma^{2} \delta+\alpha \beta^{2} \delta^{3}-\alpha \gamma^{4} \delta \\
& \left.+\alpha \gamma^{2} \delta^{3}+4 \beta^{2} \gamma^{2} \delta^{2}\right)+s_{1} s_{3}\left(\alpha^{5} \gamma-4 \alpha^{4} \gamma \delta-\alpha^{3} \beta^{2} \gamma-\alpha^{3} \gamma^{3}+6 \alpha^{3} \gamma \delta^{2}+6 \alpha^{2} \beta^{2} \gamma \delta\right. \\
& \left.+2 \alpha^{2} \gamma^{3} \delta-4 \alpha^{2} \gamma \delta^{3}-9 \alpha \beta^{2} \gamma \delta^{2}-\alpha \gamma^{3} \delta^{2}+\alpha \gamma \delta^{4}+4 \beta^{2} \gamma \delta^{3}\right)+s_{2}^{2}\left(-\alpha^{4} \beta \gamma+3 \alpha^{3} \beta \gamma \delta\right.
\end{aligned}
$$




$$
\begin{aligned}
& \left.+\alpha^{2} \beta^{3} \gamma+\alpha^{2} \beta \gamma^{3}-3 \alpha^{2} \beta \gamma \delta^{2}-\alpha \beta^{3} \gamma \delta-3 \alpha \beta \gamma^{3} \delta+\alpha \beta \gamma \delta^{3}+2 \beta \gamma^{3} \delta^{2}\right)+s_{2} s_{3}\left(\alpha^{5} \beta-4 \alpha^{4} \beta \delta\right. \\
& -\alpha^{3} \beta^{3}-\alpha^{3} \beta \gamma^{2}+6 \alpha^{3} \beta \delta^{2}+2 \alpha^{2} \beta^{3} \delta+6 \alpha^{2} \beta \gamma^{2} \delta-4 \alpha^{2} \beta \delta^{3}-\alpha \beta^{3} \delta^{2}-9 \alpha \beta \gamma^{2} \delta^{2}+\alpha \beta \delta^{4} \\
& \left.\left.+\quad 4 \beta \gamma^{2} \delta^{3}\right)+s_{3}^{2}\left(-2 \alpha^{3} \beta \gamma \delta+6 \alpha^{2} \beta \gamma \delta^{2}-6 \alpha \beta \gamma \delta^{3}+2 \beta \gamma \delta^{4}\right)\right)+m h_{+} \omega\left(s_{1}\left(-\alpha^{3} \gamma+\alpha^{2} \gamma \delta+2 \alpha \beta^{2} \gamma\right)\right. \\
& \left.+s_{2}\left(-\alpha^{3} \beta+\alpha^{2} \beta \delta+2 \alpha \beta \gamma^{2}\right)+s_{3}\left(-2 \alpha^{2} \beta \gamma+2 \alpha \beta \gamma \delta\right)+2 m^{2} h_{\times} \alpha^{2} \beta\right]+\kappa\left[h _ { + } \omega ^ { 2 } \left(s _ { 1 } ^ { 2 } \left(-\alpha^{6}\right.\right.\right. \\
& +\quad 3 \alpha^{5} \delta+2 \alpha^{4} \beta^{2}+\alpha^{4} \gamma^{2}-3 \alpha^{4} \delta^{2}-5 \alpha^{3} \beta^{2} \delta-2 \alpha^{3} \gamma^{2} \delta+\alpha^{3} \delta^{3}-\alpha^{2} \beta^{4}-\alpha^{2} \beta^{2} \gamma^{2}+4 \alpha^{2} \beta^{2} \delta^{2} \\
& \left.+\quad \alpha^{2} \gamma^{2} \delta^{2}+2 \alpha \beta^{4} \delta-\alpha \beta^{2} \delta^{3}-\beta^{4} \delta^{2}+\beta^{2} \gamma^{2} \delta^{2}\right)+s_{1} s_{2}\left(2 \alpha \beta^{3} \gamma \delta-2 \alpha \beta \gamma^{3} \delta-2 \beta^{3} \gamma \delta^{2}\right. \\
& \left.+\quad 2 \beta \gamma^{3} \delta^{2}\right)+s_{1} s_{3}\left(-\alpha^{5} \beta+4 \alpha^{4} \beta \delta+\alpha^{3} \beta^{3}+\alpha^{3} \beta \gamma^{2}-6 \alpha^{3} \beta \delta^{2}-4 \alpha^{2} \beta^{3} \delta+4 \alpha^{2} \beta \delta^{3}\right. \\
& \left.+\quad 5 \alpha \beta^{3} \delta^{2}-3 \alpha \beta \gamma^{2} \delta^{2}-\alpha \beta \delta^{4}-2 \beta^{3} \delta^{3}+2 \beta \gamma^{2} \delta^{3}\right)+s_{2}^{2}\left(\alpha^{6}-3 \alpha^{5} \delta-\alpha^{4} \beta^{2}-2 \alpha^{4} \gamma^{2}\right. \\
& +\quad 3 \alpha^{4} \delta^{2}+2 \alpha^{3} \beta^{2} \delta+5 \alpha^{3} \gamma^{2} \delta-\alpha^{3} \delta^{3}+\alpha^{2} \beta^{2} \gamma^{2}-\alpha^{2} \beta^{2} \delta^{2}+\alpha^{2} \gamma^{4}-4 \alpha^{2} \gamma^{2} \delta^{2}-2 \alpha \gamma^{4} \delta \\
& \left.+\quad \alpha \gamma^{2} \delta^{3}-\beta^{2} \gamma^{2} \delta^{2}+\gamma^{4} \delta^{2}\right)+s_{2} s_{3}\left(\alpha^{5} \gamma-4 \alpha^{4} \gamma \delta-\alpha^{3} \beta^{2} \gamma-\alpha^{3} \gamma^{3}+6 \alpha^{3} \gamma \delta^{2}+4 \alpha^{2} \gamma^{3} \delta\right. \\
& \left.-\quad 4 \alpha^{2} \gamma \delta^{3}+3 \alpha \beta^{2} \gamma \delta^{2}-5 \alpha \gamma^{3} \delta^{2}+\alpha \gamma \delta^{4}-2 \beta^{2} \gamma \delta^{3}+2 \gamma^{3} \delta^{3}\right)+s_{3}^{2}\left(\alpha^{3} \beta^{2} \delta-\alpha^{3} \gamma^{2} \delta\right. \\
& \left.\left.-\quad 3 \alpha^{2} \beta^{2} \delta^{2}+3 \alpha^{2} \gamma^{2} \delta^{2}+3 \alpha \beta^{2} \delta^{3}-3 \alpha \gamma^{2} \delta^{3}-\beta^{2} \delta^{4}+\gamma^{2} \delta^{4}\right)\right)+m \omega h_{\times}\left(s _ { 1 } \left(\alpha^{3} \beta-\alpha^{2} \beta \delta\right.\right. \\
& \left.\left.-\quad \alpha \beta^{3}+\alpha \beta \gamma^{2}\right)+s_{2}\left(-\alpha^{3} \gamma+\alpha^{2} \gamma \delta-\alpha \beta^{2} \gamma+\alpha \gamma^{3}\right)+s_{3}\left(\alpha^{2} \beta^{2}-\alpha^{2} \gamma^{2}-\alpha \beta^{2} \delta+\alpha \gamma^{2} \delta\right)\right) \\
& \left.\left.+m^{2} h_{+}\left(-\alpha^{2} \beta^{2}+\alpha^{2} \gamma^{2}\right)\right]\right\} /\left(2 m^{2} \omega \alpha^{2}(\alpha-\delta)\right)
\end{aligned}
$$

\section{References}

[1] Schutz B F 1999 Class. Quantum Grav. 16 A131

[2] Blair D G 1991 The detection of gravitational waves ed D G Blair (Cambridge University Press)

[3] Misner C W, Thorn K S and Wheeler J A 1970 Gravitation (Freeman, San Francisco)

[4] Schutz B F 1985 A first course in general relativity pp.217-19 (Cambridge University Press)

[5] van Holten J W gr-qc/9704043

[6] Grishchuk L P 1977 Sov. Phys. Usp. 20319

[7] Bini D and Gemelli G 1997 Nuovo Cimento 112 B 165

[8] Papapetrou A 1951 Proc. Roy. Soc. Lond. A209 248

[9] Pirani F A 1956 Acta Phys. Polon. 15389

[10] Moller C 1949 Comm. Dublin Ins. Adv. Stud. A 51

Tulczyjew W 1959 Acta Phys. Polon. 18393

[11] Dixon W G 1964 Nuovo Cimento 34317

[12] Dixon W G 1970 Proc. Roy. Soc. Lond. A314 499

[13] Ehlers J and Rudolph E 1977 Gen. Rel. Grav. 8197

[14] Wald R 1972 Phys. Rev D 6406

Kunzle H P 1972 J. Math. Phys. 13739

Barducci A, Casalbouni R and Lusanna L 1977 Nucl. Phys. B 124521

Ravndal F 1980 Phys. Rev. D 212823 
Yee K and Bander M 1993 Phys. Rev. D 482797

Ferrarese G, Bini D, Satzi L, Gemelli G and Ricci M 1996 Nuovo Cimento 111 B 217

[15] Cho H T 1998 Class. Quantum Grav. 152465

[16] Catenacci R and Martellini M 1977 Lett. Nuovo Cimento 20282

[17] Tod K P, De Felice F and Calvani M 1976 Nuovo Cimento 34 B 365

[18] Mohseni M, Tucker R W and Wang C 2000, submitted for bublication.

[19] Grishchuk L P and Polnarev A G 1980 General Relativity and Gravitation ed A Held pp. 393-438 (Plenum, New York)

[20] Mohseni M 2000 submitted for publication.

[21] Work in progress.

[22] These calculations have been performed using the package Excalc in REDUCE. 